Proyecciones Journal of Mathematics

Vol. 37, No 1, pp. 153-169, March 2018.

Universidad Católica del Norte

Antofagasta - Chile

\title{
Sequentially spaces and the finest locally K-convex of topologies having the same onvergent sequences
}

\author{
A. El Amrani \\ University Sidi Mohamed Ben Abdellah, Morocco \\ Received: June 201\%. Accepted : November 2017
}

\begin{abstract}
The present paper is concerned with the concept of sequentially topologies in non-archimedean analysis. We give characterizations of such topologies.
\end{abstract}

Keywords : Non-archimedean topological space, sequentially spaces, convergent sequence in non-archimedean space.

MSC2010 : $11 F 85-46 A 03-46 A 45$. 


\section{Introduction}

In 1962 Venkataramen, in [19], posed the following problem:

Characterize "the class of topological spaces which can be specified completely by the knowledge of their convergent sequences".

Several authors then agreed to provide a solution, based on the concept of sequential spaces, in particular: In [9] and [10] Franklin gave some properties of sequential spaces, examples, and a relationship with the Frechet spaces; after Snipes in [17], has studied a new class of spaces called Tsequential space and relationships with sequential spaces; in [2], Boone and Siwiec gave a characterization of sequential spaces by sequential quotient mappings; in [4], Cueva and Vinagre have studied the $\mathbf{K}-c$-Sequential spaces and the $\mathbf{K}-s$-bornological spaces and adapted the results established by Snipes using linear mappings; thereafter Katsaras and Benekas, in [13], starting with a topological vector space (t.v.s.) $(E, \tau)$, have built up, the finest of topologies on $E$ having the same convergent sequences as $\tau$; and the thinnest of topologies on $E$ having the same precompact as $\tau$; using the concept of String (this study is a generalization of the study led by Weeb [21], on 1968, in case of locally convex spaces l.c.s. ); in [8], Ferrer, Morales and Ruiz, have reproduced previous work by introducing the concept of maximal sequentially topology. Goreham, in [11], has conducted a study linking sequentiality and countable subsets in a topological space by considering the five classes of spaces: spaces of countable first case, sequential spaces, Frechet spaces, spaces of "C.T." type and perfect spaces.

In this work, we will study, in the non-archimedean (n.a) case, for a locally $\mathbf{K}$-convex space $E$ the finest sequential locally $\mathbf{K}$-convex topology on $E$ having the same convergent sequences as the original topology.

\section{Preliminaries}

Throughout this paper $\mathbf{K}$ is a (n.a) non trivially valued complete field with the valuation |.|, and the valuation ring is $B(0,1):=\{\lambda \in \mathbf{K}:|\lambda| \leq 1\}$. There exists $\varrho \in \mathbf{R}$ such that $\varrho>1$ and for all $n \in \mathbf{Z}$ there exists $\lambda_{n} \in \mathbf{K}$ verifing $\left|\lambda_{n}\right|=\varrho^{n}$ see [18],p.251.

The field $\mathbf{K}$ is spherically complete if any decreasing sequence of closed balls in $\mathbf{K}$ has a non-empty intersection.

For the basic notions and properties concerning locally $\mathbf{K}$-convex spaces we refer to [14] or [18] if $\mathbf{K}$ is spherically complete and to [15] if $\mathbf{K}$ is not 
spherically complete. However we recall the following:

Let $E$ be a $\mathbf{K}$-vector space, a nonempty subset $A$ of $E$ is called $\mathbf{K}$-convex if $\lambda x+\mu y+\gamma z \in A$ whenever $x, y, z \in A, \lambda, \mu, \gamma \in \mathbf{K},|\lambda| \leq 1$, $|\mu| \leq 1,|\gamma| \leq 1$ and $\lambda+\mu+\gamma=1$. $A$ is said to be absolutely $\mathbf{K}$-convex if $\lambda x+\mu y \in A$ whenever $x, y \in A, \lambda, \mu \in \mathbf{K},|\lambda| \leq 1,|\mu| \leq 1$. For a nonempty set $A \subset E$ its absolutely $\mathbf{K}$-convex hull $c_{0}(A)$ is the smallest absolutely $\mathbf{K}-$ convex set that contains $A$. If $A$ is a finite set $\left\{x_{1}, \ldots, x_{n}\right\}$ we sometimes write $c_{0}\left(x_{1}, \ldots, x_{n}\right)$ instead of $c_{0}(A)$.

A topology on a vector space $E$ over $\mathbf{K}$ is said to be locally $\mathbf{K}$-convex $(l \mathbf{K} c s)$ if there exists in $E$ a fundamental system of zero-neighbourhoods consisting of absolutely $\mathbf{K}$-convex subsets of $E$.

If $E$ is a $l \mathbf{K} c s, E^{\prime}$ and $E^{*}$ denote its topological and algebraic dual, respectively, and $\sigma\left(E, E^{\prime}\right)$ and $\sigma\left(E^{\prime}, E\right)$ the weak topology of $E$ and $E^{\prime}$, respectively.

If $(E, \tau)$ is a locally $\mathbf{K}$-convex space with topology $\tau$ we denote by $\mathcal{P}_{E}$, (or by $\mathcal{P}$ if no confusion is possible) a family of semi-norms determining the topology $\tau$. We always assume that $(E, \tau)$ is a Hausdorff space.

If $A$ is a subset of $E$ we denote by $[A]$ the vector space spanned by $A$. Remark that, if $A$ is absolutely $\mathbf{K}$-convex $[A]=\mathbf{K} A$. For an absolutely $\mathbf{K}$-convex subset $A$ of $E$ we denote by $p_{A}$ the Minkowski functional on $[A]$, i.e for $x \in[A], p_{A}(x)=\inf \{|\lambda|: x \in \lambda A\}$. If $A$ is bounded then $p_{A}$ is a norm on $[A]$. We then denote by $E_{A}$ the space $[A]$ normed by $p_{A}$.

Let $\langle$,$\rangle be a duality between E$ and $F$ where $E$ and $F$ are two vectors spaces over $\mathbf{K}$ (see [1] for general results), if $A$ is a subset of $E$, the polar of $A$ is a subset of $F$ defined by $A^{\circ}=\{y \in F / \forall x \in A|\langle x, y\rangle| \leq 1\}$.

We define also the polar of a subset $B$ of $F$ in the same way. A subset $A$ of $E$ is said to be a polar set if $A^{\circ \circ}=A\left(A^{\circ \circ}\right.$ is the bipolar of $\left.A\right)$

A continuous semi-norm $p$ on $E$ is called a polar seminorm if the corresponding zero-neighbourhood $A=\{x \in E: p(x) \leq 1\}$ is a polar set. The space $E$ is called strongly polar if every continuous semi-norm on $E$ is polar, and it is called polar if $\exists \mathcal{P}_{E}$ such that every $p \in \mathcal{P}_{E}$ is polar. (see [15]). Obviously:

$E$ strongly polar $\Longrightarrow E$ polar. 
If $E$ is a polar space then the weak topology $\sigma\left(E, E^{\prime}\right)$ is Hausdorff. ([15] prop. 5.6). In that case we have a dual pair $\left(E, E^{\prime}\right)$. The value of the bilinear form on $E \times E^{\prime}$ (and similarly on $E \times{ }^{\prime} E$ ) is denoted by $\langle x, a\rangle$, $x \in E, a \in E^{\prime}$. If $E$ is a polar space and $p$ is a continuous semi-norm on $E$ we denote by $E_{p}$ the vector space $E / \operatorname{Ker}(p)$ and by $\pi_{p}$ the canonical surjection $\pi_{p}: E \longrightarrow E_{p}$. The space $E_{p}$ is normed by $\left\|\pi_{p}(x)\right\|_{p}=p(x)$. Its unit ball is $\pi_{p}(U)$, with $U=\{x \in E: p(x) \leq 1\}$. Its completion is denoted by $\widehat{E_{p}}$.

\section{Sequential spaces in non-Archimedean analysis}

\subsection{Definitions and properties}

Definitions 1. 1. Let $E$ a locally $\mathbf{K}$-convex space and $V$ a subset of E.

$V$ is called a sequential neighborhood ( $S$ - neighborhood ) of 0 if every null sequence in $E$ lies eventually in $V$, that is to say:

$$
\left(\forall\left(x_{n}\right)_{n} \in C_{0}\right)\left(\exists N_{0} \in \mathbf{N}\right):\left(\forall n \geq N_{0}\right), x_{n} \in V .
$$

2. A locally $\mathbf{K}$-convex space $E$ is called sequential space if every convex sequential neighborhood of 0 is a neighborhood of 0 .

Remark 1. Every sequential neighborhood of 0 is absorbent and contains 0 .

Proposition 1. If $V$ is absolutely $\mathbf{K}$-convex and absorbent subset of a locally $\mathbf{K}$-convex space $E$, the following are equivalent:

(i) $V$ is a $S-$ neighborhood of 0 ;

(ii) $p_{V}$ is sequentially continuous. Where $p_{V}$ is the Minkowski functional associated to $V$.

Proof. $\quad(i) \Rightarrow(i i)$ Suppose that $V$ is a sequentially neighbourhood of 0 ; and let $\left(x_{n}\right)_{n} \in C_{0}(E)$, let us show that $P_{V}\left(x_{n}\right) \stackrel{n \rightarrow+\infty}{\longrightarrow} 0$.

Let $\varepsilon>0$. Let us consider $\lambda \in \mathbf{K}$ such that $0<|\lambda| \leq \varepsilon$, then $\left(\frac{x_{n}}{\lambda}\right)_{n} \in$ $C_{0}(E)$, from where there exists $N \in \mathbf{N}$ such that $(\forall n \geq N), \frac{x_{n}}{\lambda} \in V$, 
which implies that $\forall n \geq N, p_{V}\left(\frac{x_{n}}{\lambda}\right) \leq 1$, or $(\forall n \geq N), p_{V}\left(x_{n}\right) \leq|\lambda| \leq \varepsilon$. Thus the result follows.

Reciprocally, suppose that $p_{V}$ is sequentially continuous over $E$. Let $\left(x_{n}\right)_{n} \in C_{0}(E)$, so $P_{V}\left(x_{n}\right) \stackrel{n \rightarrow+\infty}{\longrightarrow} 0$, therefore there exists $N \in \mathbf{N}$ such that $(\forall n \geq N), p_{V}\left(x_{n}\right)<1$, and so $(\forall n \geq N), x_{n} \in V$.

Proposition 2. For a locally $\mathbf{K}$-convex space $E$ the following are equivalent:

(i) $E$ is a sequential space;

(ii) Every sequentially continuous seminorm on $E$ is continuous;

(iii) For every locally $\mathbf{K}$-convex space $F$, every sequentially continuous linear map from $E$ to $F$ is continuous;

(iv) For every Banach space $F$, every sequentially continuous linear map from $E$ to $F$ is continuous.

Proof. $\quad(i) \Rightarrow(i i)$. Suppose that $E$ is sequential and let $p$ a seminorm sequentially continuous on $E$. Let: $V=\{x \in E: p(x) \leq 1\}$.

$V$ is a sequential neighborhood of 0 and so $V$ is a neighborhood of 0 and consequently $p$ is continuous: $\forall \varepsilon>0$, let $\lambda \in \mathbf{K}$ such that $0<|\lambda|<\varepsilon$. Then:

$U=\lambda V$ is a neighborhood of 0 and we have $p(U) \subset B(0, \varepsilon)$.

$(i i) \Rightarrow(i)$. Let $V$ a convex subset of $E$ which is a sequential neighborhood of $0 . V$ is absorbent and contains 0 , therefore it's absolutely $\mathbf{K}$-convex $(\mathbf{K}$-convex and contains 0$)$. Then, by Proposition $1, p_{V}$ is sequetially continuous, then continuous, and so $V$ is a neighborhood of 0 . Therefore $E$ is sequential.

$($ ii $) \Rightarrow($ iii $)$. Let $F$ a locally $\mathbf{K}$-convex space and $f: E \longrightarrow F$ a sequentially-continuous linear mapping .

Let $V$ a $\mathbf{K}$-convex neighborhood of 0 in $F, f^{-1}(V)$ is a sequential $\mathbf{K}$-convex neighborhood of 0 in $E$, and so $f^{-1}(V)$ is a neighborhood of 0 in $E,(E$ is sequential and $(i i) \Leftrightarrow(i))$. And then $f$ is continuous. 
$(i v) \Rightarrow(i i)$. Let $p$ a sequentially-continuous semi-norm on $E$; consider the Banach space $\widehat{E_{p}}$ the completion of $E_{p}=E / \operatorname{Ker}(p)$. The canonical mapping $\pi_{p}: E \longrightarrow \widehat{E_{p}}$ is sequentially continuous, because: for all $\left(x_{n}\right)_{n} \subset$ $E$ such that $x_{n} \stackrel{n \rightarrow+\infty}{\longrightarrow} 0$, we have:

$$
\begin{aligned}
x_{n} \stackrel{n \rightarrow+\infty}{\longrightarrow} 0 & \Rightarrow p\left(x_{n}\right) \stackrel{n \rightarrow+\infty}{\longrightarrow} 0 \\
& \Rightarrow \widehat{p}\left(\widehat{x_{n}}\right) \stackrel{n \rightarrow+\infty}{\longrightarrow} 0 \\
& \Rightarrow \pi_{p}\left(x_{n}\right) \stackrel{n \rightarrow+\infty}{\longrightarrow} 0 .
\end{aligned}
$$

Then $\pi_{p}$ is continuous, and so $p$ is continuous:

$$
(\forall \varepsilon>0) \quad U=\pi_{p}^{-1}\left(B_{\widehat{p}}(0, \varepsilon)\right) \text { is a neighborhood of } 0 \text { in } E
$$

and we have $\mathrm{p}(U) \subset B(0, \varepsilon)$.

(iii) $\Rightarrow($ iv) Obvious.

\subsection{The sequential topology}

Let $(E, \tau)$ a locally $\mathbf{K}$-convex space. Consider $\mathcal{U}$ the set of all sequentially $\mathbf{K}$-convex neighborhood of 0 and let $\mathcal{P}_{s}$ the family of all sequentially $\tau$-continuous n.a. semi-norm on $E$.

- $\mathcal{U}$ is a base of neighborhood of 0 for a locally $\mathbf{K}$-convex topology on $E$ which is denoted $\tau^{s}[16,1.2 . p .14]$. Since every neighborhood of 0 is a sequential neighborhood of 0 , then $\tau \leq \tau^{s}$.

- $\mathcal{P}_{s}$ define a locally $\mathbf{K}$-convex topology on $E$ which is denoted $T^{s}$. A base of neighborhood of 0 for $T^{s}$ is formed by the balls $B_{p}(0, \varepsilon)$ where $\varepsilon>0$ and $p$ is a n.a. sequentially $\tau$-continuous semi-norm. $B_{p}(0, \varepsilon)$ is sequential neighborhood of 0 , because for all sequence $\left(x_{n}\right)_{n}$ converging to zero in $(E, \tau)$, there exists $n_{0} \in \mathbf{N}$ such that for all $\left(n \geq n_{0}\right), p\left(x_{n}\right)<\varepsilon\left(p\left(x_{n}\right) \stackrel{n \rightarrow+\infty}{\longrightarrow} 0\right)$.

Remark 2. The topology $T^{s}$ is sequential.

Proposition 3. $\tau^{s}$ is the coarset of all sequential locally $\mathbf{K}$-convex topologies on $E$ finer than $\tau$.

Proof. $\quad \tau^{s}$ is sequential and $\tau^{s} \geq \tau$.

Let $\varrho$ a sequential locally $\mathbf{K}$-convex topology on $E$ finer than $\tau$. Let $U \in \mathcal{U} ; U$ sequential neighborhood of 0 for $\tau$, and so $U$ is a sequential neighborhood of 0 for $\varrho(\varrho \geq \tau)$ and then $U$ is a neighborhood of 0 for $\varrho$ ( $\varrho$ is sequential). Therefore $\varrho \geq \tau^{s}$. Which proves the proposition. 


\subsubsection{Characterization of sequential locally $K$ - convex spaces}

Proposition 4. $\tau$ is sequential if, and only if, $\tau=\tau^{s}$.

Proof. $\Longleftarrow$ Obvious.

Suppose that $\tau$ is sequential and let $U \in \mathcal{U}$; $U$ is a sequential $\mathbf{K}$-convex neighborhood of 0 for $\tau$, so $U$ is a neighborhood of 0 for $\tau$ and then $\tau \geq \tau^{s}$. Finally $\tau=\tau^{s}$.

Lemma 1. For all sequence $\left(x_{n}\right)_{n}$ of $(E, \tau)$ we have:

$$
\left(x_{n} \stackrel{n \rightarrow+\infty}{\rightarrow} 0 \text { for } \tau\right) \Leftrightarrow\left(x_{n} \stackrel{n \rightarrow+\infty}{\rightarrow} 0 \text { for } \tau^{s}\right) .
$$

Proof. $\quad \Rightarrow$ Let $U \in \mathcal{U}$, there exists $N \in \mathbf{N}$ such that: $\forall n \geq N \quad x_{n} \in U$, hence $x_{n} \stackrel{n \rightarrow+\infty}{\rightarrow} 0$ for $\tau^{s}$.

The converse follows by $\tau \leq \tau^{s}$.

Lemma 2. Let $\varrho$ a locally $\mathbf{K}$-convex topology on $E$ such that for all null sequence for $\tau$ is a null sequence for $\varrho$. Then $\tau^{s} \geq \varrho$.

Proof. Consider $i:(E, \tau) \longrightarrow(E, \varrho)$ the canonical injection. Then for every sequence $\left(x_{n}\right)_{n}$ in $E$ we have:

$$
\begin{aligned}
\mathrm{x}_{n} \stackrel{\tau^{s}}{\longrightarrow} 0 \stackrel{\text { Lemma }}{\longrightarrow} x_{n} \stackrel{\tau}{\longrightarrow} 0 \\
x_{n} \stackrel{\varrho}{\longrightarrow}
\end{aligned}
$$

Then, $i$ is sequentially continuous, and since $\left(E, \tau^{s}\right)$ is sequential, $i$ is continuous (Proposition 2). Hence $\tau \leq \tau^{s}$.

Proposition 5. $\tau^{s}$ is the finest locally $\mathbf{K}$-convex topology on $E$ having the same convergent suequences as $\tau$.

Proof. By Lemma 1 before, $\tau^{s}$ and $\tau$ has the same convergent sequences. Let $\varrho$ a locally $\mathbf{K}$-convex topology on $E$ having the same convergent sequence as $\tau$ and let $\left(x_{n}\right)_{n}$ a sequence of $E$ converging to 0 for $\tau$, then $x_{n} \longrightarrow 0$ for $\varrho$, hence, by Lemma $3, \tau^{s} \geq \varrho$.

Remark 3. $\tau^{s}$ is also the finer topology on $E$ having the same null sequences as $\tau$.

Lemma 3. Let $(E, \tau)$ a locally $\mathbf{K}$-convex space and $A$ a subset of $E$, then: $A$ is $\tau$-bounded if, and only if, for all null sequence $\left(\lambda_{n}\right)_{n}$ in $\mathbf{K}$ and all sequence $\left(x_{n}\right)_{n}$ in $A$; the sequence $\left(\lambda_{n} x_{n}\right)_{n}$ converges to zero in $(E, \tau)$ that is to say $\left(\lambda_{n} x_{n}\right)_{n}$ is a null sequence in $(E, \tau)$. 
Proof. Suppose that $A$ be bounded in $(E, \tau)$.

Let $\left(\lambda_{n}\right)_{n} \in C_{0}(\mathbf{K})$ and $\left(x_{n}\right)_{n}$ a sequence in $A$.

Let $V$ a $\mathbf{K}$-convex neighborhood of zero in $E$, then there exists $\lambda$ in $\mathbf{K}^{*}$ such that $\lambda A \subset V$ and there exists $N \in \mathbf{N}^{*}$ such that $(\forall n \geq N) \quad\left|\lambda_{n}\right| \leq|\lambda|$; but

$$
\begin{aligned}
(\forall n \geq N) \quad \lambda_{n} x_{n} & =\frac{\lambda_{n}}{\lambda} \lambda x_{n} \\
& \in \frac{\lambda_{n}}{\lambda} \lambda A \\
& \subset \frac{\lambda_{n}}{\lambda} V \\
& \subset V .
\end{aligned}
$$

Then the sequence $\left(\lambda_{n} x_{n}\right)_{n}$ converges to zero in $(E, \tau)$.

Reciprocally, if $A$ is no $\tau$-bounded, then there exists $U$ a $\mathbf{K}$-convex neighborhood of zero such that $\forall n \in \mathbf{N} \quad A \not \subset \frac{1}{\lambda_{n}} U$ where $\left(\lambda_{n}\right)_{n}$ is the sequence of general term $\left|\lambda_{n}\right|=\varrho^{n}$ and $\varrho$ is the real number defined in the preliminary. For all $n \in \mathbf{N}$, let $x_{n}$ the element of $A$ such that $x_{n} \notin \frac{1}{\lambda_{n}} U$, then, $(\forall n \in \mathbf{N}) \quad \lambda_{n} x_{n} \notin U$ that is to say that the sequence $\left(\lambda_{n} x_{n}\right)_{n}$ does not converge to zero, and we have: $\left(x_{n}\right)_{n} \subset A$ and $\left(\lambda_{n}\right)_{n} \in C_{0}(\mathbf{K})$.

Proposition 6. Let $(E, \tau)$ a locally $\mathbf{K}$-convex space, then: $\tau$ and $\tau^{s}$ have the same bounded subsets.

Proof. Let $A$ a subset of $E$.

If $A$ is $\tau^{s}$-bounded, $A$ is $\tau$-bounded, because $\tau^{s} \geq \tau$.

If $A$ is $\tau$-bounded, let $\left(x_{n}\right)_{n} \subset A$ and $\left(\lambda_{n}\right)_{n} \in C_{0}(\mathbf{K})$, then, according to the previous Lemma, the sequence $\left(\lambda_{n} x_{n}\right)_{n}$ converges to zero in $(E, \tau)$ and therefore it converges to zero in $\left(E, \tau^{s}\right)$ (Lemma 1$)$. So $A$ is $\tau^{s}$-bounded.

Proposition 7. Let $\left(F, \tau^{\prime}\right)$ a locally $\mathbf{K}$-convex space and $f: E \longrightarrow F$ a linear mapping, then:

$f$ is $\tau^{s}$-continuous if, and only if, $f$ is sequentially $\tau$-continuous.

Proof. Suppose that $f$ be $\tau^{s}$-continuous, and let $\left(x_{n}\right)_{n}$ a converging sequence to zero in $(E, \tau)$ and let $V \in \mathcal{V}_{F}(0)$, there exists $U \in \mathcal{U}$ such that $f(U) \subset V$. $U$ being a sequential neighborhood of zero, so there exists $n_{0} \in \mathbf{N}$ such that $\left(\forall n \geq n_{0}\right) \quad x_{n} \in U$ and consequently $\left(\forall n \geq n_{0}\right)$ $f\left(x_{n}\right) \in f(U)(\subset V)$. Therefore the sequence $\left(f\left(x_{n}\right)\right)_{n}$ converges to zero in $F$. 
Conversely, suppose that $f$ is sequentially $\tau$-continuous; let us show that $f:\left(E, \tau^{s}\right) \longrightarrow F$ is continuous. According to Proposition 2, it suffices to show that $f$ is sequentially $\tau^{s}$-continuous. Let then $\left(x_{n}\right)_{n}$ a converging sequence to zero in $\left(E, \tau^{s}\right)$, then it converges to zero in $(E, \tau)$ (Lemma 1) and consequently $\left(f\left(x_{n}\right)\right)_{n}$ is converging to zero in $F$.

\subsection{Comparison of topologies $\tau^{s}$ and $T^{s}$}

Lemma 4. For every $U \in \mathcal{U}, p_{U}$ is a n.a. sequentially $\tau$-continuous seminorm.

Proof. Let $U \in \mathcal{U}$; then for all $\left(x_{n}\right)_{n} \in C_{0}(E)$, all $\varepsilon>0$ and all $\lambda \in \mathbf{K}^{*}$ such that $0<|\lambda| \leq \varepsilon$ we have: $\left(\lambda^{-1} x_{n}\right)_{n} \in C_{0}(E)$ from where it exists $n_{0} \in \mathbf{N}:\left(\forall n \geq n_{0}\right) \quad \lambda^{-1} x_{n} \in U$ and then:

$\left(\forall n \geq n_{0}\right) \quad p_{U}\left(\lambda^{-1} x_{n}\right) \leq 1 \Rightarrow\left(\forall n \geq n_{0}\right) \quad p_{U}\left(x_{n}\right) \leq|\lambda| \leq \varepsilon$. Therefore the sequence $\left(p_{U}\left(x_{n}\right)\right)_{n}$ converges to zero in $\mathbf{R}^{+}$and consequently $p_{U}$ is sequentially $\tau$-continuous.

Proposition 8. $\tau^{s}=T^{s}$

Proof. $\quad T^{s}$ being a sequential locally $\mathbf{K}$-convex topology (Remark 2), whence $\tau^{s} \geq T^{s}$.

For the other inequality, it suffices to show that $i:\left(E, T^{s}\right) \rightarrow\left(E, \tau^{s}\right)$ is continuous, and by Proposition2, it suffices to show that the mapping $i$ is sequentially $T^{s}$-continuous.

Let $\left(x_{n}\right)_{n}$ a sequence that tends towards zero in $\left(E, T^{s}\right)$. Then for any $U \in \mathcal{U}, p_{U}$ is sequentially $\tau$-continuous, therefore the sequence $\left(p_{U}\left(x_{n}\right)\right)_{n}$ converges to zero in $\mathbf{R}^{+}$, from where it exists $n_{0} \in \mathbf{N}:\left(\forall n \geq n_{0}\right) \quad p_{U}\left(x_{n}\right)<$ 1 , or $\left(\forall n \geq n_{0}\right) \quad x_{n} \in U$. Therefore the sequence $\left(x_{n}\right)_{n}$ tends to zero in $\left(E, \tau^{s}\right)$. From where $i$ is $T^{s}$-sequentially continuous. And consequently $T^{s} \geq \tau^{s}$.

Remark 4. We can show otherwise the previous Proposition: Since any n.a. $\tau$-continuous seminorm on $E$ is sequentially $\tau$-continuous, $T^{s} \geq \tau$. But $T^{s}$ is sequential and $\tau^{s}$ is the coarset sequential locally $\mathbf{K}$-convex topology finer than $\tau$, then $T^{s} \geq \tau^{s}$.

\subsection{The sequential polar topology}

Let $\mathcal{V}$ the family of all $\mathbf{K}$-convex, subsets $A$ of $E$ which are polar and sequential neighborhood of 0 in $(E, \tau) . \mathcal{V}$ is a base of neighborhood of 0 of 
a locally $\mathbf{K}$-convex topology on $E$ which we noted $\tau^{p s}[16,1.2 .$, p. 14$] . \tau^{p s}$ is a polar topology on $E$ and $\tau^{s} \geq \tau^{p s} \quad(\mathcal{V} \subset \mathcal{U})$.

Remark 5. Since, if $V \in \mathcal{V}$, then $\bar{V}^{\tau} \in \mathcal{V}$, we can suppose that all $V \in \mathcal{V}$, $V$ is $\tau$-closed.

Lemma 5. Suppose that $\mathbf{K}$ is spherically complete, and let $A$ a subset of $E$ absolutely $\mathbf{K}-$ convex and $\tau$-closed, then:

1. If $\mathbf{K}$ is discrete, $A^{\circ \circ}=A$.

2. If $\mathbf{K}$ is dense, $\forall \alpha \in \mathbf{K}:|\alpha|>1 A^{\circ \circ} \subset \alpha A$.

Where $A^{\circ \circ}$ is the bipolar of $A$ with respect the duality $\left\langle E, E^{\prime}\right\rangle$.

Proof. See [18, Theorems 4.14, 4.15, p.280-281].

Lemma 6. If $\mathbf{K}$ is spherically complete, then $\tau^{p s}$ is a sequential topology.

Proof. Let $U$ a subset of $E$ which is $\mathbf{K}$-convex, $\tau$-closed and sequential neighborhood of 0 on $(E, \tau)$. Let us show that $U$ is a neighborhood of 0 of $\tau^{p s}$. By Lemma 5, before $U^{\circ \circ} \subset \alpha U$ for $\alpha=1$ if $\mathbf{K}$ is discrete and $|\alpha|>1$ is $\mathbf{K}$ dense. We pose $V=U^{\circ}$, then $V$ is $\mathbf{K}$-convex, polar and sequential neighborhood of 0 on $(E, \tau)(U \subset V)$, then $V$ is a neighborhood of 0 for $\tau^{p s}$ and therefore $U$ is a neighborhood of 0 for $\tau^{p s}\left(\frac{1}{\alpha} V \subset U\right)$. Then $\tau^{p s}$ is sequential.

Proposition 9. If $\mathbf{K}$ is spherically complete, then $\tau^{p s} \geq \tau$.

Proof. It is a matter of showing that $i:\left(E, \tau^{p s}\right) \longrightarrow(E, \tau)$ is continuous, and since $\tau^{p s}$ is sequential (Lemma 6), it suffices to show that $i$ is sequentially $\tau^{p s}$-continuous.

Let $\left(x_{n}\right)_{n}$ a sequence of $E$ which is converging to zero on $\left(E, \tau^{p s}\right)$ and let $U$ an absolutely $\mathbf{K}$-convex and $\tau$-closed neighborhood of zero on $(E, \tau)$, then $U^{\circ \circ} \subset \alpha U$ where $\alpha=1$ if $\mathbf{K}$ is discrete and $|\alpha|>1$ if $\mathbf{K}$ is dense (Lemma 5). The sequence $\left(\alpha x_{n}\right)_{n}$ converges to zero on $\left(E, \tau^{p s}\right)$ and $U^{\circ \circ} \in \mathcal{V}$ hence there exists $n_{0} \in \mathbf{N}:\left(\forall n \geq n_{0}\right) \quad \alpha x_{n} \in U^{\circ \circ}$ and so $\left(\forall n \geq n_{0}\right) \quad x_{n} \in \frac{1}{\alpha} U^{\circ \circ} \subset U$. Then the sequence $\left(x_{n}\right)_{n}$ converges to zero on $(E, \tau)$. 
Remark 6. If $\mathbf{K}$ is spherically complete, then $\tau^{p s} \geq \tau$; but $\tau^{s}$ is the coarset of all sequential locally $\mathbf{K}$-convex topologies finest than $\tau$ and since $\tau^{p s}$ is sequential, then $\tau^{p s} \geq \tau^{s}$, and so $\tau^{p s}=\tau^{s}$.

Proposition 10. Let $(E, \tau)$ a locally $\mathbf{K}$-convex space. Then $\tau^{p s}$ is the finer of all polar locally $\mathbf{K}$-convex topologies $\delta$ on $E$ such that all sequence on $E$ which is $\tau$-convergent is $\delta$-convergent.

Proof. $\quad \tau^{p s}$ is a locally $\mathbf{K}$-convex polar topology on $E$.

Let $\left(x_{n}\right)_{n}$ a converging sequence to zero on $(E, \tau)$, then for all $V \in \mathcal{V}$, there exists $n_{0} \in \mathbf{N}:\left(\forall n \geq n_{0}\right) \quad x_{n} \in V$, then $\left(x_{n}\right)_{n}$ converges to zero on $\left(E, \tau^{p s}\right)$.

Let $\delta$ a locally $\mathbf{K}$-convex polar topology on $E$ such that all sequence on $E$ which is $\tau$-convergent is $\delta$-convergent; showing that $\tau^{p s} \geq \delta$. Let $U$ a $\mathbf{K}$-convex and polar neighborhood of zero for $\delta$, and let $\left(x_{n}\right)_{n}$ a sequence which converges to zero on $(E, \tau)$, then it's convergent to zero for $\delta$; hence there exists $n_{0} \in \mathbf{N}:\left(\forall n \geq n_{0}\right) \quad x_{n} \in U$. Then $U$ is a sequential neighborhood of zero and so $U \in \mathcal{V}$. And then $\tau^{p s} \geq \delta$.

Corollary 1. If $\tau$ is polar, then $\tau^{p s} \geq \tau$ and $\tau^{p s}$ and $\tau$ have the same convergent sequences.

Proof. $\quad \tau^{p s} \geq \tau$ follows immediately of the proposition before and we have all $\tau^{p s}$-convergent sequence is $\tau$-convergent. And we have already all $\tau$-convergent sequence is $\tau^{p s}$-convergent; then $\tau^{p s}$ and $\tau$ have the same convergent sequences.

Or equivalentely the two topologies have the same null sequences .

Lemma 7. Let $p$ a seminorm n.a. over E. And let:

$$
A=\{x \in E: p(x)<1\} \text { and } B=\{x \in E: p(x) \leq 1\} .
$$

Then $A^{\circ}=B^{\circ}$.

Proof. If $\mathbf{K}$ is discrete, $A=B$, then we can suppose that $\mathbf{K}$ is dense.

$A$ is a subset of $B$, then $B^{\circ} \subset A^{\circ}$. 
Let $f \in E^{*}$ such that $f \notin B^{\circ}$, then there exists $y \in B$ such that $|f(y)|>1$. Suppose that $f \in A^{\circ}$, that is to say that $(\forall x \in A) \quad|f(x)| \leq 1$; then, since $\mathbf{K}$ is dense, there exists $\lambda \in \mathbf{K}$ such that $1<|\lambda|<|f(y)|$ so:

$$
\begin{aligned}
1<\left|f\left(\frac{y}{\lambda}\right)\right| & \Longrightarrow \frac{y}{\lambda} \notin A \\
& \Longrightarrow p\left(\frac{y}{\lambda}\right) \geq 1 \\
& \Longrightarrow p(y) \geq|\lambda| \\
& \Longrightarrow p(y)>1 \\
& \Longrightarrow y \notin B
\end{aligned}
$$

Which is absurd.

Proposition 11. $\tau^{p s}$ coincides with the locally $\mathbf{K}$-convex topology generated by all n.a. polar and sequentially $\tau$-continuous semi-norms.

Proof. Let $T^{p s}$ the locally $\mathbf{K}$-convex topology generated by $\mathcal{S}_{p}$ the familly of all n.a. polar and sequentially $\tau$-continuous semi-norms. Then $T^{p s}$ admits a basis $\mathcal{B}$ of neighborhoods of zero formed by polar balls $B_{p}(0, \varepsilon)$ where $p \in \mathcal{S}_{p}$ and $\varepsilon>0$.

Let us show that $i:\left(E, \tau^{p s}\right) \longrightarrow\left(E, T^{p s}\right)$ is bicontinuous.

Let $V=B_{p}(0, \varepsilon)$ an element of $\mathcal{B}$, then $V$ is $\mathbf{K}$-convex. Let $\left(x_{n}\right)_{n}$ a sequence of elements of $E$ which converges to zero in $(E, \tau)$, then $\lim _{n \rightarrow+\infty} p\left(x_{n}\right)=0$ ( $p$ is sequetially $\tau$-continuous $)$, hence there is $n_{0} \in \mathbf{N}$ : $\left(\forall n \geq n_{0}\right) \quad p\left(x_{n}\right)<\varepsilon$, or $\left(\forall n \geq n_{0}\right) \quad x_{n} \in V$ which implies that $V$ is a sequentially neighborhood of zero, hence $V \in \mathcal{V}$ and so $T^{p s} \leq \tau^{p s}$.

Conversely, either $V \in \mathcal{V}$, then it's sequentially $\mathbf{K}$-convex neighborhood of zero. Whe have:

$$
\left\{x \in E: p_{V}(x)<1\right\} \subset V \subset\left\{x \in E: p_{V}(x) \leq 1\right\} .
$$

And by the previous Lemma 7:

$$
A^{\circ \circ}=B^{\circ \circ}=V^{\circ \circ}=V,
$$

where $A=\left\{x \in E: p_{V}(x)<1\right\}$ and $B=\left\{x \in E: p_{V}(x) \leq 1\right\}$; from where $B^{\circ \circ}=B$, and consequently $p$ is polar or $p_{V}$ is polar $[15$, Proposition $3.4, p .195]$. Let us show that $p_{V}$ is sequentially $\tau$-continuous. Let $\left(x_{n}\right)_{n}$ a sequence of elements of $E$ which converges to zero in $(E, \tau)$ and let $\varepsilon>0$; let us consider $\lambda \in \mathbf{K}$ such that $0<|\lambda|<\varepsilon$, then 
the sequence $\left(\lambda^{-1} x_{n}\right)_{n}$ converges to zero in $(E, \tau)$ and $V$ being a sequential neighborhood of 0 , then there exists $n_{0} \in \mathbf{N}:\left(\forall n \geq n_{0}\right) \quad \lambda^{-1} x_{n} \in V$, from where $\left(\forall n \geq n_{0}\right) \quad p_{V}\left(\lambda^{-1} x_{n}\right) \leq 1$ or $\left(\forall n \geq n_{0}\right) \quad p_{V}\left(x_{n}\right) \leq\left|\lambda^{-1}\right|<\varepsilon$; from where $p_{V}$ is sequentially $\tau$-continuous and consequently $p_{V} \in \mathcal{S}_{p}$; then $T^{p s} \geq \tau^{p s}$. So what $T^{p s}=\tau^{p s}$.

Proposition 12. $\tau^{p s}$ is the finer of all polar locally $\mathbf{K}$-convex topologies which are less fine than $\tau^{s}$.

Proof. $\quad \tau^{p s}$ is a polar locally $\mathbf{K}$-convex topology and $\tau^{p s} \leq \tau^{s}$.

Let $\varrho$ a polar locally $\mathbf{K}$-convex topology such that $\varrho \leq \tau^{s}$, and let $V$ a polar $\mathbf{K}$-convex neighborhood of 0 for $\varrho$, then there exists $U \in \mathcal{U}$ such that $U \subset V \quad\left(\varrho \leq \tau^{s}\right)$, from where $V$ is a sequential neighborhood of zero, consequently it is a sequential neighborhood of zero for $\tau^{p s}$. Therefore $\tau^{p s} \geq$ $\varrho$.

\subsubsection{Continuity of linear mappings}

Lemma 8. Let $E$ and $F$ be two locally $\mathbf{K}-$ convex spaces and $f: E \longrightarrow F$ a continuous linear mapping, then for any subset $V$ of $F$.

If $V$ is polar in $F, f^{-1}(V)$ is polar in $E$.

Proof. Let $V \subset F$, putting $U=f^{-1}(V)$. Suppose that $V$ is sequential.

Let $x \in U^{\circ \circ}$; let us show that $x \in U$. By absurd, suppose that $x \notin U$, and let $y=f(x)$ then $y \notin V$ from where $y \notin V^{\circ \circ}\left(V^{\circ \circ}=V\right)$ then there exists $\varphi \in V^{\circ}:|\varphi(y)|>1$. But $\forall t \in U, \quad f(t) \in V$ from where

$\forall t \in U \quad|\varphi(f(t))| \leq 1$ and consequently $\varphi \circ f \in U^{\circ}$ and so $|\varphi(f(x))| \leq$ 1 , therefore $|\varphi(y)| \leq 1$; which is absurd.

Proposition 13. Let $(E, \tau)$ and $\left(F, \tau_{1}\right)$ two locally $\mathbf{K}$-convex spaces .

If $f:(E, \tau) \longrightarrow\left(F, \tau_{1}\right)$ is a continuous linear mapping, then $f$ is $\left(\tau^{s}, \tau_{1}^{s}\right)$-continuous and $\left(\tau^{p s}, \tau_{1}^{p s}\right)$-continuous.

Proof. Let us show that $f:\left(E, \tau^{s}\right) \longrightarrow\left(F, \tau_{1}^{s}\right)$ is continuous. For this it suffices to show that for every sequential neighborhood $V$ of zero for 
$\tau_{1}, f^{-1}(V)$ is a sequential neighborhood $V$ of zero for $\tau$.

Let $V$ a sequential neighborhood of zero for $\tau_{1}$ and let $\left(x_{n}\right)_{n}$ a sequence of $E$ which converges towards zero in $(E, \tau)$; then the sequence $\left(f\left(x_{n}\right)\right)_{n}$ converges towards zero in $\left(F, \tau_{1}\right)$, from where there exists $n_{0} \in$ $\mathbf{N}:\left(\forall n \geq n_{0}\right) f\left(x_{n}\right) \in V$, then $\left(\forall n \geq n_{0}\right) x_{n} \in f^{-1}(V)$.

Let us show that $f:\left(E, \tau^{p s}\right) \longrightarrow\left(F, \tau_{1}^{p s}\right)$ is continuous. For this it suffices to show that for every polar and sequential neighborhood $V$ of zero for $\tau_{1}, f^{-1}(V)$ is a polar and sequential neighborhood $V$ of zero for $\tau$.

Let $V$ a polar and sequential neighborhood of zero for $\tau_{1}$, then by Lemma $8, f^{-1}(V)$ is polar for $\tau$. In the other hand, for all sequence $\left(x_{n}\right)_{n}$ of $E$ which converges towards zero in $(E, \tau)$, the sequence $\left(f\left(x_{n}\right)\right)_{n}$ converges to zero in $\left(F, \tau_{1}\right)$, from where there exists $n_{0} \in \mathbf{N}:\left(\forall n \geq n_{0}\right) f\left(x_{n}\right) \in V$, therefore $\left(\forall n \geq n_{0}\right) x_{n} \in f^{-1}(V)$.

Proposition 14. Let $(E, \tau)=\prod_{k=1}^{n}\left(E_{k}, \tau_{k}\right)$, then:

(i) $\tau^{s}=\prod_{k=1}^{n} \tau_{k}^{s}$;

(ii) $\tau^{p s}=\prod_{k=1}^{n} \tau_{k}^{p s}$.

Proof. Let us show that $i:\left(E, \tau^{s}\right) \longrightarrow\left(E, \prod_{k=1}^{n} \tau_{k}^{s}\right)$ is continuous. Let us show that $V$ is neighborhood of zero for $\left(E, \tau^{s}\right)$, where $V=\left(U_{k}\right)_{1 \leq k \leq n}$ is a $K$-convex neighborhood of zero for the arrival space. Let $\left(y_{p}\right)_{p}=\left(\prod_{k=1}^{n} x_{k}^{p}\right)_{p}$ a sequence of $E$ which converges to zero in $(E, \tau)$, then for all $k \in \mathbf{N}, 1 \leq k \leq n$, the sequence $\left(x_{k}^{p}\right)_{p}$ converges to zero in $\left(E_{k}, \tau_{k}\right)$, from where there exists $p_{k} \in \mathbf{N}: \quad\left(\forall p \geq p_{k}\right) \quad x_{k}^{p} \in U_{k}$. Let $p_{0}=\max _{1 \leq k \leq n} p_{k}$, so $\left(\forall p \geq p_{0}\right) \forall k \in \mathbf{N}, 1 \leq k \leq n, \quad x_{k}^{p} \in U_{k}$, from where $\left(\forall p \geq p_{0}\right) \quad y_{p} \in V$. Therefore $V$ is a sequential neighborhood of zero in $(E, \tau) ; V$ being $\mathbf{K}$-convex, therefore $V$ is a neighborhood of zero in $\left(E, \tau^{s}\right)$. 
Let us show that $i:\left(E, \prod_{k=1}^{n} \tau_{k}^{s}\right) \longrightarrow\left(E, \tau^{s}\right)$ is continuous. Let $V$ a sequential $\mathbf{K}$-convex neighborhood of zero in $(E, \tau)$. For all $k, 1 \leq k \leq n$, let $j_{k}: E_{k} \rightarrow E$ the canonical injection and pose $V_{k}=j_{k}^{-1}(V)$, so $V_{k}$ is a sequential neighborhood of zero in $\left(E_{k}, \tau_{k}\right)$, from where $V_{k}$ is a neighborhood of zero in $\left(E_{k}, \tau_{k}^{s}\right)$, and consequently $U=\prod_{k=1}^{n} V_{k}$ is a neighborhood of zero in $\left(E, \prod_{k=1}^{n} \tau_{k}^{s}\right)$. But $U \subset V(V$ is absolutely $\mathbf{K}-$ convex $)$; therefore $V$ is a neighborhood of zero in $\prod_{k=1}^{n} \tau_{k}^{s}$.

\section{References}

[1] R. Ameziane Hassani and M. Babahmed, Topologies polaires compatibles avec une dualité séparante sur un corps valué non-archimédien, Proyecciones. Vol. 20, No. 2, pp. 217-241, (2001).

[2] J. R. Boone and F.Siwiec, Sequentially quotient mappings, Czechoslovak Mathematical Journal, Vol. 26, No. 2, pp. 174-182, (1976).

[3] J. Boos and T. Leiger, Duals pairs of sequence spaces, IJMMS 28, No.1, pp. 9-23, (2001).

[4] M. C. Cueva and C. T. Maia Vinagre, Sequences in non-archimedean locally convex spaces, Indian J. pure appl. Math., 25(9): pp. 955-962, (1994).

[5] N. De Grande-De Kimpe, Perfect locally K-convex sequence spaces, Indag. Math. (33), pp. 371-482, (1971).

[6] A. El amrani, R. Ameziane Hassani and M. Babahmed, Topologies on sequence spaces in non-archimedean analysis, J. of Mathematical Sciences: Advances and Applications 6, No. 2, pp. 193-214, (2010).

[7] A. El Amrani, R. Ameziane Hassani and M. Babahmed, Polar topologies in sequence spaces in non-archimedean analysis. Universidad Catolica del Norte Antofagasta-Chile. Proyecciones Journal of Mathematics 31, No. 2, pp. 103-123, (2012). 
[8] J. R. Ferrer, I. Morales, L. M. Sanchez Ruiz, Sequential convergence in topological vector spaces, Topology and its applications 108, pp. 1-6, (2000).

[9] S. P. Franklin, Spaces in which sequences suffice," Fundamenta Mathematicae 67, pp. 107-116, (1965).

[10] S. P. Franklin, "Spaces of which sequences suffice, II," Fund. Math. 61, pp. 51-56, (1967).

[11] A. Goreham, Sequential convergence in topological spaces, arXiv:math/0412558v2-[math.GN] 10 April, (2016).

[12] D.A. Gregory, Vector sequence spaces, Ph.D thesis, University of Machigan, (1967).

[13] A. K. Katsaras and V. Benekas, Sequential convergence in topological vector spaces, Georgian mathematical journal: Vol. 2, No. 2, pp. 151$164,(1995)$.

[14] A. F. Monna, Analyse non- archimédienne, Springer-Verlag, band 56, (1970).

[15] W. H. Schikhof, Locally convex spaces over nonspherically complete valued field, I, II, Bull. Soc. Math. Belg. sér. B 38, pp. 187-224 (1986).

[16] H. H. Schaefer, Topological vector spaces, Springer-Verlag New-York, herdlberg Berlin, (1971).

[17] R. F. Snipes, T-sequential topological spaces, Fundamenta Mathematicae, T. LXXVII, pp. 95-98, (1970).

[18] J. Van-tiel, Espaces localement $K$-convexes, I-III. Proc. Kon. Ned. Akad. van Wetensch. A 68, pp. 249-289, (1965).

[19] M. Venkataramen, Directed sets in topology, Math. student 30, pp. 99-100, (1962).

[20] S. Warner, Topological Fields, North-Holland Mathematics studies 157, (1989).

[21] J. H. Webb, Sequential convergence in locally convex spaces, Proc. Cambridge Philos. Soc. 64, pp. 341-364, (1968). 
Sequentially spaces and the finest locally $\mathbf{K}$-convex of topologies... 169

\author{
A. El Amrani \\ Department of mathematics and computer science \\ Faculty of Sciences Dhar El Mahraz \\ B. P. 1796 Atlas Fès, \\ Morocco \\ e-mail: abdelkhalek.elamrani@usmba.ac.ma
}

